\title{
The Equivariant Generating Hypothesis
}

\author{
ANNA MARIE BOHMANN
}

\begin{abstract}
We state the generating hypothesis in the homotopy category of $G$-spectra for a compact Lie group $G$ and prove that if $G$ is finite, then the generating hypothesis implies the strong generating hypothesis, just as in the non-equivariant case. We also give an explicit counterexample to the generating hypothesis in the category of rational $S^{1}$-equivariant spectra.
\end{abstract}

$55 \mathrm{P} 91 ; 55 \mathrm{P} 42$

\section{The generating hypothesis in an equivariant context}

The Freyd conjecture, also known as the Freyd generating hypothesis, is a long-standing conjecture in stable homotopy theory. Let $\mathrm{HoS}$ be the homotopy category of spectra and let $\pi_{*}(S)$-mod be the category of $\pi_{*}(S)$-modules, where $S$ is the sphere spectrum.

Conjecture 1.1 (Generating hypothesis) The restriction of the functor

$$
\pi_{*}(-): \operatorname{Ho} \mathcal{S} \rightarrow \pi_{*}(S)-\bmod
$$

to the subcategory of finite spectra is faithful. That is, if a map $f: X \rightarrow Y$ between finite spectra $X$ and $Y$ induces the zero map $f_{*}: \pi_{*}(X) \rightarrow \pi_{*}(Y)$, then $f$ is nullhomotopic.

This conjecture was introduced by Freyd in 1965 [4] and remains open today. Recent work has examined analogous statements in algebraic categories that share many properties of the homotopy category of spectra, such as the derived category of a ring (see Hovey et al [7] or Lockridge [10]) or the stable module category of a finite group (see Benson et al [1] or Carlson et al [2]). Here we examine the analogous conjecture for the homotopy category of $G$-equivariant spectra for a compact Lie group $G$.

The appropriate generalization of the generating hypothesis to an equivariant context must take into account the homotopy groups of the fixed-point spectra for each closed subgroup $H \leq G$. This is best formulated in the language of Mackey functors. Let $\mathcal{M}[G]$ be the category of Mackey functors over a compact Lie group $G$, let $\mathcal{M}[G]_{\text {* }}$ be the category of graded Mackey functors, and let HoGS be the homotopy category of $G$-spectra. 
Conjecture 1.2 (Equivariant generating hypothesis) The restriction of the equivariant homotopy functor $\underline{\pi}_{*}^{G}(-): \operatorname{Ho} G \mathcal{S} \rightarrow \mathcal{M}[G]_{*}$ to the subcategory of finite $G$-spectra is faithful. That is, if a map $f: X \rightarrow Y$ between finite $G$-spectra $X$ and $Y$ induces the zero map $f_{*}: \underline{\pi}_{*}^{G}(X) \rightarrow \underline{\pi}_{*}^{G}(Y)$, then $f$ is nullhomotopic.

A map $M \rightarrow N$ of Mackey functors is zero if and only if the map $M(G / H) \rightarrow N(G / H)$ is zero for each closed subgroup $H \leq G$. Recall that the Mackey functor $\underline{\pi}_{n}^{G}(X)$ is given at $G / H$ by

$$
\underline{\pi}_{n}^{G}(X)(G / H)=\left[S^{n} \wedge G / H_{+}, X\right]^{G}=\pi_{n}^{H}(X) .
$$

Hence this conjecture can also be stated as requiring a map $f: X \rightarrow Y$ of finite $G-$ spectra to be nullhomotopic if it induces the zero map $f_{*}: \pi_{*}^{H}(X) \rightarrow \pi_{*}^{H}(Y)$ for all closed subgroups $H \leq G$. Since the conjecture does not make use of the full structure of a Mackey functor, we will not give an explicit definition here but instead refer the reader to Dress [3].

Remark 1.3 The non-equivariant generating hypothesis can be recovered as a special case of the equivariant generating hypothesis by taking $G$ to be the trivial group; however, there is no immediate implication between the non-equivariant generating hypothesis and the equivariant generating hypothesis for any other group of equivariance. In particular, since the Lewis-May fixed-point spectrum of a finite $G$-spectrum need not be finite, the non-equivariant generating hypothesis does not apply to the maps $X^{H} \rightarrow Y^{H}$ induced by an equivariant map $X \rightarrow Y$.

The above formulation of the Freyd Conjecture (Conjecture 1.1) is also known as the weak generating hypothesis, as opposed to the strong generating hypothesis. Freyd's original formulation of the strong generating hypothesis conjectures that the functor $\pi_{*}(-)$ is both faithful and full when restricted to the subcategory of finite spectra. In his initial paper on the subject, Freyd also proves the following relationship between the weak and strong generating hypotheses.

Theorem 1.4 (Freyd [4, Proposition 9.7]) If the weak generating hypothesis 1.1 holds, then so does the strong generating hypothesis. That is, if the restriction of the functor $\pi_{*}(-)$ : $\operatorname{Ho} \mathcal{S} \rightarrow \pi_{*}(S)-\bmod$ to the subcategory of finite spectra is faithful, $\pi_{*}(-)$ is also full on this subcategory.

This type of "faithful implies full" implication does not always hold, as shown by Hovey, Lockridge, and Puninski in the case of the derived category of a ring [7]. However, this implication is true in the equivariant case for a finite group $G$. 
Theorem 1.5 Let $G$ be a finite group, and let $\mathcal{M}[G]_{*}$ be the category of graded Mackey functors over $G$. Then, if the functor $\underline{\pi}_{*}^{G}(-): \operatorname{HoGS} \rightarrow \mathcal{M}[G]_{*}$ is faithful on restriction to the full subcategory of finite spectra, $\underline{\pi}_{*}^{G}(-)$ is also full on this subcategory.

We also consider the generating hypothesis in the category of rational $G$-spectra. For a finite group $G$, Greenlees and May [6, Appendix A] have proved that there is a natural equivalence between rational $G$-spectra and the category of graded Mackey functors over $\underline{A} \otimes \mathbb{Q}$, where $\underline{A}$ is the Burnside ring Mackey functor. This equivalence is induced by the natural isomorphism

$$
\vartheta:\left[X_{0}, Y_{0}\right]^{G} \rightarrow \prod_{n} \operatorname{Hom}_{\mathcal{M}[G]}\left(\underline{\pi}_{n}^{G}\left(X_{0}\right), \underline{\pi}_{n}^{G}\left(Y_{0}\right)\right)
$$

for rational $G$-spectra $X_{0}$ and $Y_{0}$. This isomorphism completely algebraicizes the rational $G$-stable homotopy category, and thus in particular establishes that the strong generating hypothesis holds in this category. As an aside, we note that the isomorphism (1-6) is also used in proving Theorem 1.5.

For infinite compact Lie groups, the picture is quite different. We prove that the weak generating hypothesis fails rationally in the simplest case of a non-finite group of equivariance, that is, for the category of rational $\mathbb{T}$-spectra, where $\mathbb{T}$ is the compact Lie group $S^{1}$.

Theorem 1.7 The equivariant generating hypothesis (Conjecture 1.2) does not hold for the category of rational $\mathbb{T}$-equivariant spectra.

This result suggests that the generating hypothesis should not hold for other infinite groups of equivariance, and it also makes immaterial the question of whether the weak generating hypothesis implies the strong generating hypothesis in the category of rational $\mathbb{T}$-equivariant spectra.

We prove Theorem 1.5 in Section 3. As a technical tool for this proof, in Section 2 we introduce the construction of an abelian envelope of a triangulated category. In Section 4, we establish Theorem 1.7 by giving a specific counterexample to the generating hypothesis in the category of rational $\mathbb{T}$-spectra. The structure of the proof of Theorem 1.7 is due in part to John Greenlees, and the author would like to thank him for his guidance and conversation. The author would also like to thank her advisor, Peter May, for his guidance and support. 


\section{The abelian envelope of a triangulated category}

Our proof of Theorem 1.5 follows Freyd's proof that the weak generating hypothesis implies the strong generating hypothesis for the category of spectra [4, Proposition 9.7]. In particular, we make use of the abelian category $\mathcal{A}(G)$ associated to the equivariant stable homotopy category. This is a special case of the general construction of an abelian envelope of a triangulated category. This construction was first given by Freyd [4]; for a more modern treatment, including several equivalent constructions, see Chapter 5 of Neeman's book on triangulated categories [11].

Given a triangulated category $\mathcal{T}$, its abelian envelope $\mathcal{A}(\mathcal{T})$ is an abelian category with a full and faithful inclusion $\iota: \mathcal{T} \rightarrow \mathcal{A}(\mathcal{T})$ with the properties that

- for any object $s$ in $\mathcal{T}$, its image $\iota(s)$ in $\mathcal{A}(\mathcal{T})$ is projective and injective, and every projective or injective object in $\mathcal{A}(\mathcal{T})$ is a direct summand of an object in the image of $\iota$,

(2-1) - this construction is self-dual, in that $\mathcal{A}\left(\mathcal{T}^{\mathrm{op}}\right) \cong \mathcal{A}(\mathcal{T})^{\mathrm{op}}$, and

- any homological functor $H: \mathcal{T} \rightarrow \mathcal{A}$, where $\mathcal{A}$ is an abelian category, extends uniquely to an additive functor $\mathcal{A}(\mathcal{T}) \rightarrow \mathcal{A}$.

By homological functor, we mean a functor that takes exact triangles of $\mathcal{T}$ to exact sequences in $\mathcal{A}$. The self-duality of the construction implies that a cohomological functor $H: \mathcal{T}^{\mathrm{op}} \rightarrow \mathcal{A}$ extends to a unique contravariant functor $\mathcal{A}\left(\mathcal{T}^{\mathrm{op}}\right)=\mathcal{A}(\mathcal{T})^{\mathrm{op}} \rightarrow \mathcal{A}$ taking exact sequences to exact sequences.

We define $\mathcal{A}(\mathcal{T})$ to be the following quotient of the category of maps in $\mathcal{T}$; this is Freyd's original description [4, Section 3] and is also given by Neeman [11, Definition 5.2.1].

Definition 2.2 The objects of $\mathcal{A}(\mathcal{T})$ are maps $s \rightarrow t$ in $\mathcal{T}$, and morphisms in $\mathcal{A}(\mathcal{T})$ are commutative diagrams

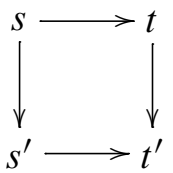

under the additive equivalence relation defined by setting such a morphism equal to zero if the composite $s \rightarrow t \rightarrow t^{\prime}$ or $s \rightarrow s^{\prime} \rightarrow t^{\prime}$ is zero. The embedding $\iota: \mathcal{T} \rightarrow \mathcal{A}(\mathcal{T})$ is given by sending an object $s$ to the identity morphism $s \rightarrow s$.

We can think of the functor $\iota$ as a universal homological functor from $\mathcal{T}$ to an abelian category. Because $\iota$ is full and faithful, we will usually identify an object $s \in \mathcal{T}$ with 
its image under $\iota$. For a proof that $\mathcal{A}(\mathcal{T})$ has the desired properties $(2-1)$, see [11, Chapter 5].

In order to make use of this construction in proving Theorem 1.5 , we need to show that if a version of the generating hypothesis holds in a triangulated category $\mathcal{T}$, it also holds in the category $\mathcal{A}(\mathcal{T})$. Recall that an object $X$ in a triangulated category $\mathcal{T}$ is compact if for any family of objects $\left\{Y_{i}\right\}$, the natural map $\bigoplus \mathcal{T}\left(X, Y_{i}\right) \rightarrow \mathcal{T}\left(X, \amalg Y_{i}\right)$ is an isomorphism.

Lemma 2.3 Let $\mathcal{T}$ be a triangulated category, let $\mathcal{A}$ be an abelian category, and let $H: \mathcal{T} \rightarrow \mathcal{A}$ be a homological functor. If $H$ is faithful on restriction to the category of compact objects of $\mathcal{T}$, then its extension to $\mathcal{A}(\mathcal{T})$ is also faithful on restriction to objects with a projective resolution by compact objects of $\mathcal{T}$.

Proof Objects in $\mathcal{A}(\mathcal{T})$ with a projective resolution by compact objects of $\mathcal{T}$ are the same as objects $s \rightarrow t$ where $s$ and $t$ are compact objects of $\mathcal{T}$ [11, Chapter 5]. The value of a homological functor $H$ on an object $s \rightarrow t$ in $\mathcal{A}(\mathcal{T})$ is given by the image in $\mathcal{A}$ of the map $H(s) \rightarrow H(t)$. If $s, t, s^{\prime}$ and $t^{\prime}$ are compact objects of $\mathcal{T}$ and we have a morphism

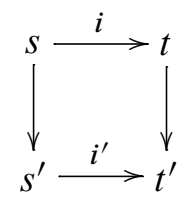

in $\mathcal{A}(\mathcal{T})$, then by definition the map $H(s \rightarrow t) \rightarrow H\left(s^{\prime} \rightarrow t^{\prime}\right)$ is zero if and only if the induced map $\operatorname{im} H(i) \rightarrow \operatorname{im} H\left(i^{\prime}\right)$ is zero. But if this is the case, then the map $H(s) \rightarrow H\left(s^{\prime}\right) \rightarrow H\left(t^{\prime}\right)$ is zero. Hence, assuming $H$ is faithful on compact objects of $\mathcal{T}$, the map $s \rightarrow s^{\prime} \rightarrow t^{\prime}$ is zero, and so our original morphism is also zero.

This lemma says that in any triangulated category where a version of the generating hypothesis holds, the generating hypothesis extends to the abelian envelope of that category. We will make use of this result in the case $\mathcal{T}=\operatorname{HoGS}$ in the next section.

\section{The proof of Theorem 1.5: Faithful implies full}

We now prove that for a finite group $G$, the generating hypothesis for $G$-spectra implies the strong generating hypothesis for $G$-spectra. Let $\mathcal{A}(G)$ be the abelian envelope of the triangulated category $\mathrm{HoG} \mathcal{S}$ as discussed in Section 2. In particular, $\mathcal{A}(G)$ is an abelian category with a full embedding $\operatorname{HoGS} \rightarrow \mathcal{A}(G)$ such that the image of every $G$-spectrum is both projective and injective. Since the embedding is full and faithful, we identify the group of maps $\mathcal{A}(G)(X, Y)$ with $[X, Y]^{G}$ for $G$-spectra $X$ and $Y$. 
Proof of Theorem 1.5 Let $G$ be a finite group. Let $X$ and $Y$ be finite $G-\mathrm{CW}$ spectra, and let $S$ be the sphere $G$-spectrum. Let $\underline{\pi}_{*}^{G}(S)$ be the graded Green functor that is $\underline{\pi}_{n}^{G}(S)$ in degree $n$, meaning that $\underline{\pi}_{*}^{G}(S)$ is a graded Mackey functor with a compatible ring structure. Note that $\underline{\pi}_{0}^{G}(S)$ is the Burnside ring Green functor. Similarly, let $\underline{\pi}_{*}^{G}(X)$ be the graded Mackey functor that is $\underline{\pi}_{n}^{G}(X)$ in degree $n$. Composition makes $\underline{\pi}_{*}^{G}(X)$ a module over $\underline{\pi}_{*}^{G}(S)$.

Suppose we have a natural transformation $\phi: \underline{\pi}_{*}^{G}(X) \rightarrow \underline{\pi}_{*}^{G}(Y)$. To prove that $\underline{\pi}_{*}^{G}(-)$ is full, we must find a map $f: X \rightarrow Y$ such that $\underline{\pi}_{*}^{G}(f)=\phi$. The structure of the proof is in two steps. First, we use the embedding $\iota: \operatorname{Ho} G \mathcal{S} \rightarrow \mathcal{A}(G)$ to construct a sequence of maps $\left\{f_{n}: X \rightarrow Y\right\}$ such that $\left(f_{n}\right)_{*}: \underline{\pi}_{j}^{G}(X) \rightarrow \underline{\pi}_{j}^{G}(Y)$ agrees with $\phi$ for $j \leq n$. Lemma 3.1 then shows that $\left\{f_{n}\right\}$ has a constant cofinal subsequence whose value is then the desired map $f$.

For each conjugacy class of subgroups $H \leq G$ and each $j$, choose a set of generators of $\underline{\pi}_{j}^{G}(X)(G / H)=\left[\Sigma^{j}\left(S \wedge G / H_{+}\right), X\right]^{G}$. Denote these generators by

$$
\alpha_{j, H, i}: \Sigma^{j}\left(S \wedge G / H_{+}\right) \rightarrow X .
$$

Define a spectrum $W_{n}$ by

$$
W_{n}=\bigvee_{j \leq n} \bigvee_{H \leq G} \bigvee_{i} \Sigma^{j}\left(S \wedge G / H_{+}\right) .
$$

Since $X$ is a finite spectrum, its homotopy groups are finitely generated and thus $W_{n}$ is a finite spectrum as well. Let $\alpha_{n}: W_{n} \rightarrow X$ be the map given on wedge summands by $\alpha_{j, H, i}$. Similarly, let $h_{n}$ : $W_{n} \rightarrow Y$ be the map given on wedge summands by $\phi\left(\alpha_{j, H, i}\right)$.

Let $\iota_{n}: K_{n} \rightarrow W_{n}$ be the kernel of the map $\alpha_{n}: W_{n} \rightarrow X$ in $\mathcal{A}(G)$. Thus, the sequence $0 \rightarrow K_{n} \rightarrow W_{n} \rightarrow X$ is exact in $\mathcal{A}(G)$. We first show that $\underline{\pi}_{*}^{G}\left(h_{n} \circ \iota_{n}\right)=0$. Then we apply the assumption that $\underline{\pi}_{*}^{G}(-)$ is faithful to conclude that $h_{n} \circ \iota_{n}$ is zero. By construction, and because $\phi$ is a natural transformation of Mackey functors,

$$
\begin{aligned}
\underline{\pi}_{*}^{G}\left(h_{n} \circ \iota_{n}\right) & =\underline{\pi}_{*}^{G}\left(h_{n}\right) \circ \underline{\pi}_{*}^{G}\left(\iota_{n}\right) \\
& =\underline{\pi}_{*}^{G}\left(\phi\left(\alpha_{n}\right)\right) \circ \underline{\pi}_{*}^{G}\left(\iota_{n}\right) \\
& =\phi\left(\underline{\pi}_{*}^{G}\left(\alpha_{n}\right)\right) \circ \underline{\pi}_{*}^{G}\left(\iota_{n}\right) \\
& =\phi\left(\underline{\pi}_{*}^{G}\left(\alpha_{n} \circ \iota_{n}\right)\right) .
\end{aligned}
$$

Since $\alpha_{n} \circ \iota_{n}=0$ in $\mathcal{A}(G)$, we see that $\underline{\pi}_{*}^{G}\left(h_{n} \circ \iota_{n}\right)=\phi\left(\underline{\pi}_{*}^{G}\left(\alpha_{n} \circ \iota_{n}\right)\right)=0$. We are assuming the generating hypothesis holds in $\operatorname{Ho} G \mathcal{S}$, so we can apply Lemma 2.3 to conclude that $h_{n} \circ \iota_{n}: K_{n} \rightarrow W_{n} \rightarrow Y$ is zero in $\mathcal{A}(G)$. 
Now we construct our maps $f_{n}$. Let $\zeta_{n}: W_{n} \rightarrow D_{n}$ be the cokernel of the map $\iota_{n}: K_{n} \rightarrow W_{n}$. Note that, as $K_{n}$ is the kernel of $\alpha_{n}: W_{n} \rightarrow X$, the map $\alpha_{n}: W_{n} \rightarrow X$ factors through an injection $v_{n}: D_{n} \rightarrow X$. Since $h_{n} \circ \iota_{n}=0$, the map $h_{n}$ factors through a map $\gamma_{n}: D_{n} \rightarrow Y$. Also let $\mu_{n}: X \rightarrow C_{n}$ be the cokernel of $\alpha_{n}: W_{n} \rightarrow X$. The object $Y$ is injective in the category $\mathcal{A}(G)$, as mentioned in (2-1), so the map $\gamma_{n}: D_{n} \rightarrow Y$ extends to a map $f_{n}: X \rightarrow Y$ such that $f_{n} \circ v_{n}=\gamma_{n}$. We summarize these definitions in the following commutative diagram in $\mathcal{A}(G)$.

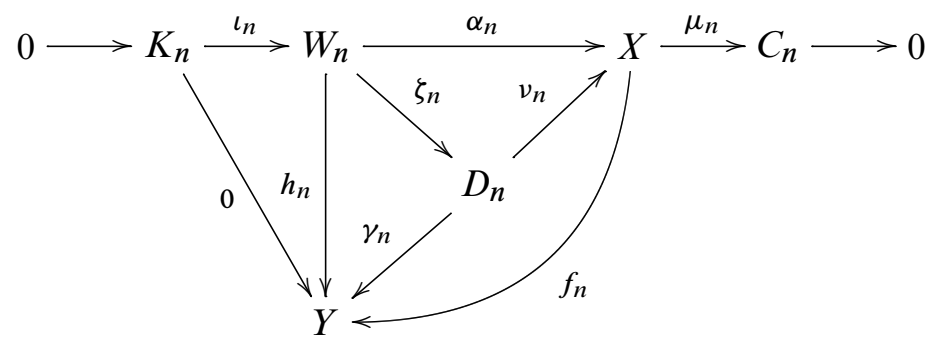

We have constructed $\alpha_{n}$ such that $\underline{\pi}_{*}^{G}\left(\alpha_{n}\right)$ is a surjection $\underline{\pi}_{j}^{G}\left(W_{n}\right) \rightarrow \underline{\pi}_{j}^{G}(X)$ for $j \leq n$. Since $\underline{\pi}_{*}^{G}\left(f_{n}\right) \circ \underline{\pi}_{*}^{G}\left(\alpha_{n}\right)=\underline{\pi}_{*}^{G}\left(h_{n}\right)=\phi\left(\underline{\pi}_{*}^{G}\left(\alpha_{n}\right)\right)$, it follows that $\underline{\pi}_{j}^{G}\left(f_{n}\right)=\phi$ for $j \leq n$. We need to construct a map $f=f_{\infty}$ that has this property for all $n$.

By construction, we have an inclusion $i_{n}: W_{n} \rightarrow W_{n+1}$ such that $\alpha_{n}=\alpha_{n+1} \circ i_{n}$ and $h_{n}=h_{n+1} \circ i_{n}$. For any $m>n$, iterating these inclusions of summands gives an inclusion $W_{n} \rightarrow W_{m}$ which makes the diagram

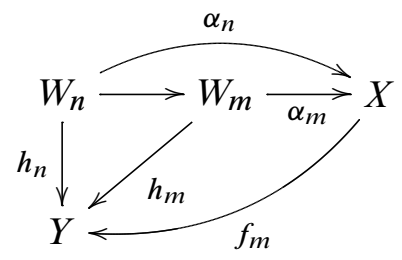

commute. Hence the restriction of the map $f_{m} \circ \alpha_{m}: W_{m} \rightarrow X \rightarrow Y$ to the summands contained in $W_{n}$ is the map $h_{n}$; that is, $f_{m} \circ \alpha_{n}=h_{n}$. By the universal property of the cokernel $\mu_{n}: X \rightarrow C_{n}$, we obtain a factorization of the map $f_{m}-f_{n}$ through $C_{n}$.

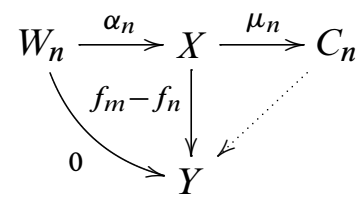

Since $X$ is finite, we may choose $d$ large enough so that $H_{j}^{G}(X ; \underline{A})=0$ for all $j>d$, where $\underline{A}$ is the Burnside ring Mackey functor for the group $G$ and $H_{*}^{G}$ is Bredon 
homology. We claim that $\mathcal{A}(G)\left(C_{d}, Y\right)$ is finite, or equivalently, since $\mathcal{A}(G)\left(C_{d}, Y\right)$ is finitely generated, that the group $\mathcal{A}(G)\left(C_{d}, Y\right) \otimes \mathbb{Q}=0$. This will complete the proof.

Lemma 3.1 Let $X, Y, W_{d}$ and $C_{d}$ be as above. Then the group $\mathcal{A}(G)\left(C_{d}, Y\right) \otimes \mathbb{Q}$ is zero.

Proof First consider $\underline{H}_{*}^{G}\left(C_{d} ; \underline{A}\right)$. Since the functor $\underline{H}_{j}^{G}(-; \underline{A}): \mathcal{A}(G) \rightarrow \mathcal{M}[G]$ is exact, for each $H \leq G$ we have an exact sequence of abelian groups

$$
\underline{H}_{j}^{G}\left(W_{d} ; \underline{A}\right)(G / H) \stackrel{\left(\alpha_{d}\right)_{*}}{\longrightarrow} \underline{H}_{j}^{G}(X ; \underline{A})(G / H) \rightarrow \underline{H}_{j}^{G}\left(C_{d} ; \underline{A}\right)(G / H) \rightarrow 0
$$

for each $j$. When $j>d$, the Mackey functor $\underline{H}_{j}^{G}(X ; \underline{A})$ is zero, and thus $\underline{H}_{j}^{G}\left(C_{d} ; \underline{A}\right)$ is zero as well. As May and Greenlees show [6, Appendix A], the rationalization of the sphere $G$-spectrum is the Eilenberg-Maclane $G$-spectrum $H(\underline{A} \otimes \mathbb{Q})$. Thus we can equate the rationalization of the sequence (3-2) with the sequence

$$
\underline{\pi}_{j}^{G}\left(W_{d}\right) \otimes \mathbb{Q} \stackrel{\left(\alpha_{d}\right)_{*}}{\longrightarrow} \underline{\pi}_{j}^{G}(X) \otimes \mathbb{Q} \rightarrow \underline{\pi}_{j}^{G}\left(C_{d}\right) \otimes \mathbb{Q} \rightarrow 0 .
$$

When $j \leq d$, the map $\alpha_{d}$ is surjective on rational homotopy by construction, so $\underline{\pi}_{j}^{G}\left(C_{d}\right) \otimes \mathbb{Q}=0$ for $j \leq d$. Since $\underline{\pi}_{j}^{G}\left(C_{d}\right) \otimes \mathbb{Q}=\underline{H}_{j}^{G}\left(C_{d} ; \underline{A}\right) \otimes \mathbb{Q}=0$ for $j>d$, the graded Mackey functor $\underline{\pi}_{*}^{G}\left(C_{d}\right) \otimes \mathbb{Q}$ is zero.

Denote by $Z_{0}$ the rationalization of a $G$-spectrum $Z$; then

$$
\left[Z, Z^{\prime}\right]^{G} \otimes \mathbb{Q}=\left[Z_{0}, Z_{0}^{\prime}\right]^{G}=\left[Z, Z_{0}^{\prime}\right]^{G} .
$$

By restricting to the category of rational $G$-spectra, we in principal get an abelian category $\mathcal{A}_{0}(G)$ for rational stable homotopy as in the construction of Definition 2.2. However, the rationalization functor $\operatorname{Ho} G \mathcal{S} \rightarrow \operatorname{Ho} G \mathcal{S}_{0}$ induces an extension $\mathcal{A}(G) \rightarrow \mathcal{A}_{0}(G)$. This functor is an equivalence after tensoring with $\mathbb{Q}$; this does not change the category $\mathcal{A}_{0}(G)$ since $\mathcal{A}_{0}(G)$ is already rational. Hence we do not distinguish between $\mathcal{A}(G) \otimes \mathbb{Q}$ and $\mathcal{A}_{0}(G)$.

For $G$-spectra $Z$ and $Z^{\prime}$, we restate the isomorphism (1-6) in terms of tensoring with $\mathbb{Q}$ :

$$
\vartheta:\left[Z, Z^{\prime}\right]^{G} \otimes \mathbb{Q} \rightarrow \prod_{n} \operatorname{Hom}_{\mathcal{M}[G]}\left(\underline{\pi}_{n}^{G}(Z) \otimes \mathbb{Q}, \underline{\pi}_{n}^{G}\left(Z^{\prime}\right) \otimes \mathbb{Q}\right) .
$$

Moreover, for a fixed $Z^{\prime}$, both sides of (3-3) are cohomology theories on $G$-spectra, so this isomorphism extends to $\mathcal{A}(G)$. Setting $Z^{\prime}=Y$ then allows us to conclude 
that the group $\mathcal{A}(G)\left(C_{d}, Y\right) \otimes \mathbb{Q}$ is zero. More precisely, the columns are exact in the following diagram.

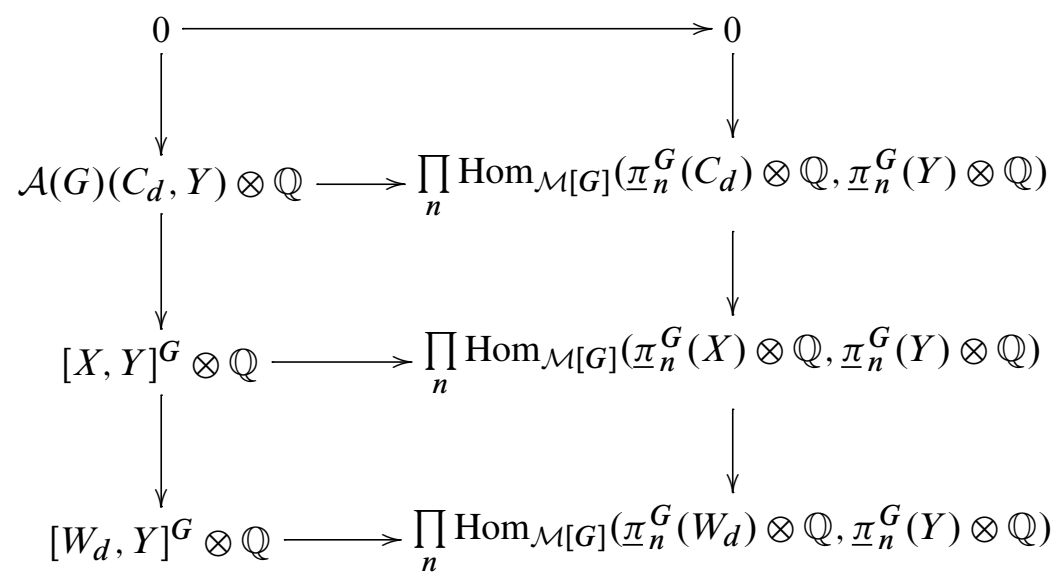

Here we are identifying $\mathcal{A}(G)(Z, Y)$ with $[Z, Y]^{G}$ for a $G$-spectrum $Z$. By (3-3), the bottom two rows are isomorphisms and hence the five lemma implies

$$
\mathcal{A}(G)\left(C_{d}, Y\right) \otimes \mathbb{Q} \cong \prod_{n} \operatorname{Hom}_{\mathcal{M}[G]}\left(\underline{\pi}_{n}^{G}\left(C_{d}\right) \otimes \mathbb{Q}, \underline{\pi}_{n}^{G}(Y) \otimes \mathbb{Q}\right) .
$$

But $\underline{\pi}_{*}^{G}\left(C_{d}\right) \otimes \mathbb{Q}=0$, so in fact $\mathcal{A}(G)\left(C_{d}, Y\right) \otimes \mathbb{Q}=0$.

Returning to our proof of Theorem 1.5, Lemma 3.1 shows that $\mathcal{A}(G)\left(C_{d}, Y\right)$ is a finite group. Therefore, the sequence $\left\{f_{n}: C_{d} \rightarrow Y\right\}$ has a constant cofinal subsequence, and we can take its value to be the desired map $f: X \rightarrow Y$ for which $\underline{\pi}_{*}^{G}(f)=\phi$.

Thus, for a finite group $G$, the weak generating hypothesis implies the strong generating hypothesis, just as in the non-equivariant case.

\section{The rational equivariant case}

Restricting to the category of rational $G$-spectra for a group $G$ greatly simplifies the structure of the category, and after this simplification the rational $G$-stable homotopy categories for finite and infinite compact Lie groups exhibit very different behavior. As mentioned in the introduction, the strong generating hypothesis holds in the rational stable homotopy category for a finite group $G$, but even the weak generating hypothesis fails in the category of $\mathbb{T}$-spectra, where $\mathbb{T}$ is the circle group. In fact, using Greenlees's algebraic model for the category of rational $\mathbb{T}$-spectra, we find an explicit counterexample to the generating hypothesis in this category. 
Theorem 1.7 The generalized Freyd conjecture does not hold for the category of rational $\mathbb{T}$-equivariant spectra.

Before proving this theorem, we prove the following lemma about the equivariant homotopy groups of the suspension spectrum $\mathbb{X}=\Sigma_{+}^{\infty} X$ of a free $\mathbb{T}$-space $X$.

Lemma 4.1 Let $X$ be a free rational $\mathbb{T}$-space, and let $\mathbb{X}=\Sigma_{+}^{\infty} X$ denote the suspension spectrum of $X_{+}$. Then, for a closed subgroup $H \leq \mathbb{T}$,

$$
\pi_{*}^{H}(\mathbb{X})= \begin{cases}\pi_{*}(\Sigma \mathbb{X} / \mathbb{T}) & \text { if } H=\mathbb{T} \\ \pi_{*}(\mathbb{X}) & \text { if } H<\mathbb{T} .\end{cases}
$$

Proof The tom Dieck splitting theorem [9] states that for such an $\mathbb{X}$ and for any closed subgroup $H \leq \mathbb{T}$,

$$
\pi_{*}^{H}(\mathbb{X})=\bigoplus_{K \leq H} \pi_{*}\left(\Sigma^{\infty}\left(E W_{H} K_{+} \wedge_{W_{H} K} \Sigma^{\operatorname{ad}\left(W_{H} K\right)} X_{+}^{K}\right)\right),
$$

where the sum runs over conjugacy classes of closed subgroups $K \leq H$ and $\operatorname{ad}\left(W_{H} K\right)$ is the adjoint representation of the Weyl group $W_{H} K$. Let us first consider $\pi_{*}^{\mathbb{T}}(\mathbb{X})$. Since $X$ is free, the space $X_{+}^{K}$ is a point unless $K$ is the trivial group. Hence the only summand that contributes to $\pi_{*}^{\mathbb{T}}(\mathbb{X})$ is the summand corresponding to the trivial group $1 \leq \mathbb{T}$. The Weyl group of $1 \leq \mathbb{T}$ is $\mathbb{T}$ itself, and the adjoint representation of $\mathbb{T}$ is trivial. Thus

$$
\pi_{*}^{\mathbb{T}}(\mathbb{X})=\pi_{*}\left(\Sigma^{\infty}\left(E \mathbb{T}_{+} \wedge_{\mathbb{T}} \Sigma X_{+}\right)\right)=\pi_{*}(\Sigma \mathbb{X} / \mathbb{T}) .
$$

Now suppose $H$ is a proper closed subgroup of $\mathbb{T}$. Then $H$ is finite, so $\operatorname{ad}\left(W_{H} K\right)$ is zero for all $K \leq H$. Since $X$ is free, the summand of (4-2) corresponding the trivial group is the only summand contributing to $\pi_{*}^{H}(\mathbb{X})$, just as in the previous case. Hence $\pi_{*}^{H}(\mathbb{X})$ reduces to

$$
\pi_{*}^{H}(\mathbb{X})=\pi_{*}\left(\Sigma^{\infty}\left(E H_{+} \wedge_{H} X_{+}\right)\right)=\pi_{*}(\mathbb{X} / H) .
$$

Since we are working rationally, $\pi_{*}(\mathbb{X} / H)=H_{*}(\mathbb{X} / H)$. As $H$ is necessarily finite, $H_{*}(\mathbb{X} / H)=H_{*}(\mathbb{X}) / H$. This follows because rationally the homology theories given by $H_{*}(-/ H)$ and $H_{*}(-) / H$ agree on orbits $H / K$, and thus must be isomorphic [8, page 29]. However, since $H$ is acting as a subgroup of the connected group $\mathbb{T}, H$ must in fact act trivially on $H_{*}(\mathbb{X})$. Thus $\pi_{*}(\mathbb{X} / H)=H_{*}(\mathbb{X})=\pi_{*}(\mathbb{X})$, where the second equality again follows by rationality. That is, for any proper closed subgroup $H \leq \mathbb{T}$, the $H$-homotopy groups $\pi_{*}^{H}(\mathbb{X})$ are in fact the non-equivariant homotopy groups $\pi_{*}(\mathbb{X})$. 
Proof of Theorem 1.7 We restrict our attention to free rational $\mathbb{T}$-spectra and use the results of Greenlees in [5]. Throughout the following proof, we assume that all spectra are rational without explicitly indicating this in our notation.

Let $\mathbb{X}$ and $\mathbb{Y}$ be finite rational $\mathbb{T}$-spectra. The equivariant Freyd conjecture asserts that a map $f: \mathbb{X} \rightarrow \mathbb{Y}$ is zero if and only if it induces the zero map on $\pi_{*}^{H}(-)$ for all closed subgroups $H \leq \mathbb{T}$. We give a counterexample where $\mathbb{X}$ and $\mathbb{Y}$ happen to be free rational $\mathbb{T}$-spectra.

Greenlees proves [5, Theorem 3.1.1] that for free rational $\mathbb{T}$-spectra, there is a natural Adams short exact sequence

$$
0 \rightarrow \operatorname{Ext}_{\mathbb{Q}[c]}\left(\pi_{*}^{\mathbb{T}}(\Sigma \mathbb{X}), \pi_{*}^{\mathbb{T}}(\mathbb{Y})\right) \rightarrow[\mathbb{X}, \mathbb{Y}]_{*}^{\mathbb{T}} \rightarrow \operatorname{Hom}_{\mathbb{Q}[c]}\left(\pi_{*}^{\mathbb{T}}(\mathbb{X}), \pi_{*}^{\mathbb{T}}(\mathbb{Y})\right) \rightarrow 0,
$$

where $\mathbb{Q}[c]$ is the graded polynomial algebra on an element $c$ in dimension -2 . This element $c$ comes from the Euler class of the representation of $\mathbb{T}$ on $\mathbb{C}$ by multiplication [5, Theorem 2.4.1]. Note that Hom and Ext are graded, and Ext here means Ext ${ }^{1, *}$. This short exact sequence is our main tool in finding a counterexample to the equivariant Freyd conjecture.

Let $X$ and $Y$ be free rational $\mathbb{T}$-spaces, and let $\mathbb{X}=\Sigma_{+}^{\infty} X$ and $\mathbb{Y}=\Sigma_{+}^{\infty} Y$. Consider the space $\mathbb{T}_{+} \wedge X_{+}$with $\mathbb{T}$ acting diagonally. There is an isomorphism

$$
\left[\Sigma^{\infty}\left(\mathbb{T}_{+} \wedge X_{+}\right), \mathbb{Y}\right]_{*}^{\mathbb{T}} \cong[\mathbb{X}, \mathbb{Y}]_{*}
$$

between equivariant homotopy classes of maps from $\Sigma^{\infty}\left(\mathbb{T}_{+} \wedge X_{+}\right)$to $\mathbb{Y}$ and nonequivariant homotopy classes of maps from $\mathbb{X}$ to $\mathbb{Y}$, and since $\mathbb{X}$ and $\mathbb{Y}$ were assumed to be rational, $[\mathbb{X}, \mathbb{Y}]_{*}$ is also isomorphic to $\operatorname{Hom}_{\mathbb{Q}}\left(\pi_{*}(\mathbb{X}), \pi_{*}(\mathbb{Y})\right)$.

Write $\mathbb{Q}[c]=k$ and $\Sigma^{\infty}\left(\mathbb{T}_{+} \wedge X_{+}\right)=\mathbb{W}$ for conciseness. The projection $p: \mathbb{W} \rightarrow \mathbb{X}$ induces a map of short exact sequences

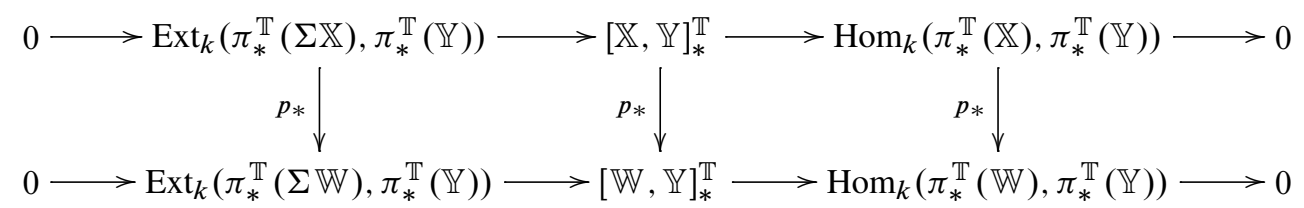

which can be rewritten as

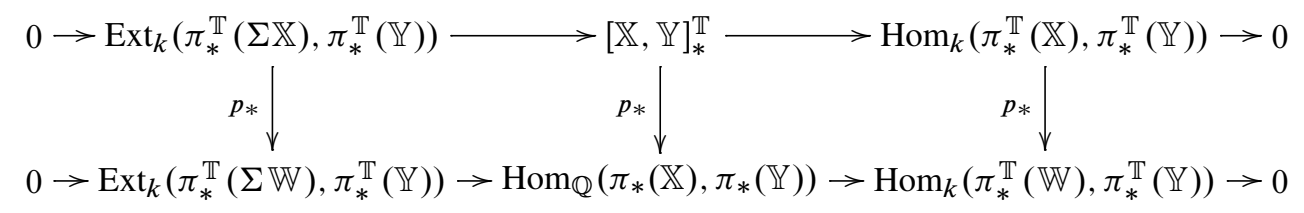


Let $f: \mathbb{X} \rightarrow \mathbb{Y}$ be a map inducing the zero map $\pi_{*}^{H}(\mathbb{X}) \rightarrow \pi_{*}^{H}(\mathbb{Y})$ for all subgroups $H \leq \mathbb{T}$. By Lemma 4.1 this is equivalent to assuming that $f_{*}: \pi_{*}^{\mathbb{T}}(\mathbb{X}) \rightarrow \pi_{*}^{\mathbb{T}}(\mathbb{Y})$ and $f_{*}: \pi_{*}(\mathbb{X}) \rightarrow \pi_{*}(\mathbb{Y})$ are both zero. In other words, $p_{*}(f)$ is zero in

$$
\operatorname{Hom}_{\mathbb{Q}}\left(\pi_{*}(\mathbb{X}), \pi_{*}(\mathbb{Y})\right)=\operatorname{Hom}_{\mathbb{Q}}\left(\pi_{*}^{H}(\mathbb{X}), \pi_{*}^{H}(\mathbb{Y})\right)
$$

and also $f$ maps to zero in $\operatorname{Hom}_{k}\left(\pi_{*}^{\mathbb{T}}(\mathbb{X}), \pi_{*}^{\mathbb{T}}(\mathbb{Y})\right)$. Hence $f$ is either zero, or lifts to a nontrivial element of $\operatorname{Ext}_{k}\left(\pi_{*}^{\mathbb{T}}(\Sigma \mathbb{X}), \pi_{*}^{\mathbb{T}}(\mathbb{Y})\right)$. To show that the generating hypothesis fails for the category of rational $\mathbb{T}$-spectra, it is enough to show that the map

$$
\operatorname{Ext}_{k}\left(\pi_{*}^{\mathbb{T}}(\Sigma \mathbb{X}), \pi_{*}^{\mathbb{T}}(\mathbb{Y})\right) \rightarrow \operatorname{Ext}_{k}\left(\pi_{*}^{\mathbb{T}}(\Sigma \mathbb{W}), \pi_{*}^{\mathbb{T}}(\mathbb{Y})\right)
$$

has a nontrivial kernel. Any element of this kernel must be nonzero as an element of $[\mathbb{X}, \mathbb{Y}]_{*}^{\mathbb{T}}$, but must map to zero in the group $[\mathbb{W}, \mathbb{Y}]_{*}^{\mathbb{T}}$ by definition, and will map to zero in $\operatorname{Hom}_{k}\left(\pi_{*}^{\mathbb{T}}(\mathbb{X}), \pi_{*}^{\mathbb{T}}(\mathbb{Y})\right)$ by exactness. Thus such an element gives a counterexample to the generating hypothesis.

We now give a concrete example where the map (4-3) has a nontrivial kernel. The group $\mathbb{T}$ acts on the complex plane $\mathbb{C}$ by multiplication. Consider the diagonal action of $\mathbb{T}$ on $\mathbb{C}^{a}$, for some positive integer $a$. Let $S(a)$ be the unit sphere in this representation of $\mathbb{T}$ and consider the rational suspension spectrum of $S(a)_{+}$. We will denote this spectrum by $\mathbb{S}(a)$. We claim that for integers $a, b>1$, the spectra $\mathbb{S}(a)$ and $\mathbb{S}(b)$ provide a counterexample to the equivariant generating hypothesis.

To prove this claim, it suffices to calculate the groups

$$
\operatorname{Ext}_{\mathbb{Q}[c]}\left(\pi_{*}^{\mathbb{T}}(\Sigma \mathbb{S}(a)), \pi_{*}^{\mathbb{T}}(\mathbb{S}(b))\right) \text { and } \operatorname{Ext}_{\mathbb{Q}[c]}\left(\pi_{*}^{\mathbb{T}}(\Sigma \mathbb{W}), \pi_{*}^{\mathbb{T}}(\mathbb{S}(b))\right)
$$

where now $\mathbb{W}=\Sigma^{\infty}\left(\mathbb{T}_{+} \wedge S(a)_{+}\right)$. First note that Lemma 4.1 implies $\pi_{*}^{\mathbb{T}}(\mathbb{S}(a))=$ $\pi_{*}(\Sigma \mathbb{S}(a) / \mathbb{T})$. Since $\mathbb{S}(a)$ is the suspension spectrum of $S(a)_{+}$and we are working rationally, we may pass to the space level to see that

$$
\pi_{*}(\Sigma \mathbb{S}(a) / \mathbb{T})=\pi_{*}\left(\Sigma \mathbb{C} P^{a-1}\right)=\Sigma^{2 a-1} \mathbb{Q}[c] /\left(c^{a}\right)
$$

as a graded module over $\mathbb{Q}[c]$. Similarly, Lemma 4.1 implies $\pi_{*}^{\mathbb{T}}(\mathbb{W})=\pi_{*}(\Sigma \mathbb{S}(a))$, and passing to the space level yields

$$
\pi_{*}(\Sigma \mathbb{S}(a))=\pi_{*}\left(\Sigma S^{2 a-1}\right)=\Sigma \mathbb{Q} \oplus \Sigma^{2 a} \mathbb{Q},
$$

again as a graded module over $\mathbb{Q}[c]$. Hence our Ext calculations reduce to finding the groups

$$
\operatorname{Ext}_{\mathbb{Q}[c]}\left(\Sigma^{2 a} \mathbb{Q}[c] /\left(c^{a}\right), \Sigma^{2 b-1} \mathbb{Q}[c] /\left(c^{b}\right)\right)
$$

and

$$
\operatorname{Ext}_{\mathbb{Q}[c]}\left(\Sigma^{2} \mathbb{Q} \oplus \Sigma^{2 a+1} \mathbb{Q}, \Sigma^{2 b-1} \mathbb{Q}[c] /\left(c^{b}\right)\right) .
$$


To calculate the first group, we use the free resolution

$$
0 \rightarrow \mathbb{Q}[c] \stackrel{c^{a}}{\rightarrow} \Sigma^{2 a} \mathbb{Q}[c] \rightarrow \Sigma^{2 a} \mathbb{Q}[c] /\left(c^{a}\right) \rightarrow 0
$$

of $\Sigma^{2 a} \mathbb{Q}[c] /\left(c^{a}\right)$. Applying $\operatorname{Hom}_{\mathbb{Q}[c]}\left(-, \Sigma^{2 b-1} \mathbb{Q}[c] /\left(c^{b}\right)\right)$ to this resolution gives an exact sequence

$$
\begin{array}{rl}
\cdots \rightarrow \Sigma^{2 b-1-2 a} \mathbb{Q}[c] /\left(c^{b}\right) \stackrel{c^{a}}{\rightarrow} \Sigma^{2 b-1} & \mathbb{Q}[c] /\left(c^{b}\right) \\
& \rightarrow \operatorname{Ext}_{\mathbb{Q}[c]}\left(\Sigma^{2 a} \mathbb{Q}[c] /\left(c^{a}\right), \Sigma^{2 b-1} \mathbb{Q}[c] /\left(c^{b}\right)\right) \rightarrow 0
\end{array}
$$

so that

$$
\operatorname{Ext}_{\mathbb{Q}[c]}\left(\Sigma^{2 a} \mathbb{Q}[c] /\left(c^{a}\right), \Sigma^{2 b-1} \mathbb{Q}[c] /\left(c^{b}\right)\right)= \begin{cases}\Sigma^{2 b-1} \mathbb{Q}[c] /\left(c^{a}\right) & \text { if } a \leq b \\ \Sigma^{2 b-1} \mathbb{Q}[c] /\left(c^{b}\right) & \text { if } a>b .\end{cases}
$$

To calculate $\operatorname{Ext}_{\mathbb{Q}[c]}\left(\pi_{*}^{\mathbb{T}}(\Sigma \mathbb{W}), \pi_{*}^{\mathbb{T}}(\mathbb{S}(b))\right)$, we use the free resolution

$$
0 \rightarrow \mathbb{Q}[c] \oplus \Sigma^{2 a-1} \mathbb{Q}[c] \rightarrow \Sigma^{2} \mathbb{Q}[c] \oplus \Sigma^{2 a+1} \mathbb{Q}[c] \rightarrow \Sigma^{2} \mathbb{Q} \oplus \Sigma^{2 a+1} \mathbb{Q} \rightarrow 0
$$

of $\Sigma^{2} \mathbb{Q} \oplus \Sigma^{2 a+1} \mathbb{Q}$. Applying $\operatorname{Hom}\left(-, \Sigma^{2 b-1} \mathbb{Q}[c] /\left(c^{b}\right)\right)$ to this sequence gives the exact sequence

$$
\begin{array}{rl}
\cdots \rightarrow \Sigma^{2 b-3} \mathbb{Q}[c] /\left(c^{b}\right) \oplus \Sigma^{2 b-2 a-2} \mathbb{Q}[c] /\left(c^{b}\right) & \\
\rightarrow \Sigma^{2 b-1} & \mathbb{Q}[c] /\left(c^{b}\right) \oplus \Sigma^{2 b-2 a} \mathbb{Q}[c] /\left(c^{b}\right) \\
\rightarrow \operatorname{Ext}_{\mathbb{Q}[c]}\left(\Sigma^{2} \mathbb{Q} \oplus \Sigma^{2 a+1} \mathbb{Q}, \Sigma^{2 b-1} \mathbb{Q}[c] /\left(c^{b}\right)\right) \rightarrow 0
\end{array}
$$

Hence

$$
\operatorname{Ext}_{\mathbb{Q}[c]}\left(\Sigma^{2} \mathbb{Q} \oplus \Sigma^{2 a+1} \mathbb{Q}, \Sigma^{2 b-1} \mathbb{Q}[c] /\left(c^{b}\right)\right)=\Sigma^{2 b-1} \mathbb{Q} \oplus \Sigma^{2 b-2 a} \mathbb{Q} .
$$

For any positive integer $n$, the set of maps from $\Sigma^{2 b-1} \mathbb{Q}[c] /\left(c^{n}\right)$ to $\Sigma^{2 b-1} \mathbb{Q} \oplus$ $\Sigma^{2 b-2 a} \mathbb{Q}$ has a $\mathbb{Q}$-basis given by two non-zero maps, one that takes the generator of $\Sigma^{2 b-1} \mathbb{Q}(c) /\left(c^{n}\right)$ to degree $2 b-1$ and one that takes this generator to degree $2 b-2 a$. If $n \geq 2$, neither of these maps is injective. Hence, for $a, b>1$, the map (4-3) cannot be injective. Any nontrivial element of the kernel of (4-3) yields a nontrivial map $\mathbb{S}(a) \rightarrow \mathbb{S}(b)$ that induces the trivial map on homotopy. Therefore $\mathbb{S}(a)$ and $\mathbb{S}(b)$ provide the desired counterexample to the equivariant generating hypothesis for the category of rational $\mathbb{T}$-spectra.

Remark 4.4 The rational Burnside ring of a compact Lie group $G$ is a von Neumann regular ring. As such, a theorem of Hovey, Lockridge and Puninski [7] implies that the appropriate formulation of the generating hypothesis in the derived category of this ring 
holds. This contrasts with our Theorem 1.7, which suggests that generating hypothesis should fail in the category of $G$-equivariant spectra. Despite the formal analogy of the statements, the generating hypothesis in the derived category of the Burnside ring of $G$ is not germane to the generating hypothesis for the $G$-equivariant homotopy category.

\section{References}

[1] D J Benson, S K Chebolu, J D Christensen, J Mináč, The generating hypothesis for the stable module category of a p-group, J. Algebra 310 (2007) 428-433 MR2307802

[2] J F Carlson, S K Chebolu, J Mináč, Freyd's generating hypothesis with almost split sequences, Proc. Amer. Math. Soc. 137 (2009) 2575-2580 MR2497468

[3] A W M Dress, Contributions to the theory of induced representations, from: "Algebraic $K$-theory, II: "Classical" algebraic $K$-theory and connections with arithmetic (Proc. Conf., Battelle Memorial Inst., Seattle, Wash., 1972)”, Springer, Berlin (1973) 183-240. Lecture Notes in Math., Vol. 342 MR0384917

[4] P Freyd, Stable homotopy, from: "Proc. Conf. Categorical Algebra (La Jolla, Calif., 1965)”, Springer, New York (1966) 121-172 MR0211399

[5] J P C Greenlees, Rational $S^{1}$-equivariant stable homotopy theory, Mem. Amer. Math. Soc. 138 (1999) MR1483831

[6] J P C Greenlees, J P May, Generalized Tate cohomology, Mem. Amer. Math. Soc. 113 (1995) MR1230773

[7] M Hovey, K Lockridge, G Puninski, The generating hypothesis in the derived category of a ring, Math. Z. 256 (2007) 789-800 MR2308891

[8] I Kř́iž, JP May, Operads, algebras, modules and motives, Astérisque (1995) MR1361938

[9] L G Lewis, Jr, J P May, M Steinberger, J E McClure, Equivariant stable homotopy theory, Lecture Notes in Mathematics 1213, Springer, Berlin (1986) MR866482 With contributions by $\mathrm{J} \mathrm{E}$ McClure

[10] K H Lockridge, The generating hypothesis in the derived category of $R$-modules, $\mathbf{J}$. Pure Appl. Algebra 208 (2007) 485-495 MR2277690

[11] A Neeman, Triangulated categories, Annals of Mathematics Studies 148, Princeton University Press, Princeton, NJ (2001) MR1812507

Department of Mathematics, University of Chicago

Chicago IL 60637, USA

bohmann@math.uchicago.edu

http://math.uchicago.edu/ bohmann/

Received: 29 July 2009 Revised: 11 February 2010 